\title{
The Possible Dimension, Additional to Space-Time, which Physicists Ignore
}

\author{
Arie S. Issar, Shaul Sorek \\ Ben-Gurion University of the Negev Israel, Blaustein Institutes for Desert Research, Israel \\ Zuckerberg Institute for Water Research, Environmental Hydrology \& Microbiology \\ E-mail: $\{$ issar, sorek\}@bgu.ac.il \\ Received February 23, 2010; revised March 25, 2010; accepted March 25, 2010
}

\begin{abstract}
In response to Wheeler's challenge to find an element that is: "something that itself has no localization in space and time...pure knowledge ... an atom of information" we suggest to account for Information as a dimension. Its degrees of freedom are arithmetical (+-) and logical (if-then) forward and backward steps. While Space refers to gaps in distance, Time refers to change in instances, Information refers to a sequence of notions measured by the number of steps made (or bytes used) by a computer in order to perform (describe or solve) a certain logical sentence or a sequence of logical sentences. In the attempt to quantifiably formulate the incorporation of Information into physical laws, we refer to Hamiltonian extended stationary principle in terms of Space, Time and an additional degree of freedom, suggested as an information state. The obtained Euler equation is demonstrated for the case of a thin rod under longitudinal vibrations, investigated by dimensionless analysis. It is shown that depending on the value of information and its rate, one may obtain dominant forms conforming to Poison's equation in Space vs Information, wave equation in Time vs Information and the expected wave equation in Time vs Space.
\end{abstract}

Keywords: Information, Hamiltonian, Dimensionless-Analysis

\section{The New Dimension Wheeler Foresaw: Information}

"If we're ever going to find an element of nature that explains space and time, we surely have to find something that is deeper than space and time-something that itself has no localization in space and time. The amazing feature of the elementary quantum phenomenon-the Great Smoky Dragon-is exactly this. It is indeed something of a pure knowledge-theoretical character, an atom of information, which has no localization in between the point of entry and the point of registration. This is the significance of the delayed-choice experiment." [1].

Following Wheeler's call [1] we maintain that Information should be (along which intelligence is measured as well as instinctive knowledge) added, independently, to space and time. At first it was thought by Issar to call this dimension ${ }^{1}$ either thinking or 'intelligence', but after investigating these options it was found that thinking is

\footnotetext{
${ }^{1}$ An intrinsic independent property, representing the minimum number of directions needed to specify either a point on space, an instant on time and a notion when considering Information.
}

the description of moving and 'intelligence' are structures along degrees of freedom along a more substantial dimension. The introduction of a new basic dimension will enable to describe intelligence as a feature or even a structure constructed from the addition of fundamental steps of observation and logical conclusion. These, are in fact the basic steps of arithmetic addition (and subtraction) and the basic logical conclusion of 'if-then', forward and backwards. Generalizing this suggestion will say that the adding one to another of a few basic conclusions, which brings to the arriving of a more general structure, is actually a construction along a dimension, which constitutes 4 degrees of freedom. The more intelligent is the living being, the more will be its ability to increase the building composed of elaborate structures of knowledge added one to another and put together by logical steps, to form wider and higher structures of intelligent thinking and behavior.

This conclusion brought to search for an altogether new dimension along which the movement while taking these fundamental steps can be described and quantified. After investigating various options the conclusion was that the most appropriate term will be: 'Information'. Thus, change in location on space is quantified by steps 
of the foot or by a meter, duration of time is quantified by the movement of shade on the sun-dial or by a clock, while progress on the Dimension of Information is quantified by the length of mathematical or logical sentences needed to describe a certain idea or number of bytes used by a computer to perform a certain logical sentence.

Space has six degrees of freedom namely along or spins around three coordinates, in two directions i.e. forward and backwards, Time one degree of freedom from present to future (the perspective from present to past actually refers to knowledge and thus is regarded as the Dimension of Information), while the additional dimension of Information has four degrees of freedom [2], which are: addition and subtraction and induction and deduction (i.e. 'if-then' and 'when-then'). Altogether the evolution of the universe is along a three dimensional continuum of Space-Time-Information, having 11 degrees of freedom.

The introduction of this new dimension enables to answer a question that many outstanding physicists came up with and which Albert Einstein [3] brought up in his lecture before the Prussian Academy of Sciences, namely: "How can it be that mathematics, being after all a product of human thought which is independent of experience, is so admirably appropriate to the objects of reality? His answer to this enigma was by accepting as a fact that Mathematics is intrinsic both in Nature and in the human mind. Yet while the human mind can build wonderful logical structures with the aid of this mysterious tool, these structures are not factual if not cross-checked by empirical observations. In Einstein's words: “In my opinion the answer to this question is, briefly, this: as far as the propositions of mathematics refer to reality, they are not certain; and as far as they are certain, they do not refer to reality [3].

In his Herbert Spencer lecture at Oxford, Einstein [4] manifested his faith in mathematics as the skeleton of the edifice of nature, and thus the power of abstract mathematical thought to reveal the secrets of the laws interconnecting our observation of natural phenomena. He stressed the role of mathematics as a bridge between mind and nature. In his words: "Our experience hitherto justifies us in believing that nature is the realization of the simplest conceivable mathematical ideas. I am convinced that we can discover by means of purely mathematical constructions the concepts and the laws connecting them with each other, which furnish the key to the understanding of natural phenomena. Useful mathematical concepts may well be suggested by experience, but in no way can they be derived from it. Experience naturally remains the sole criterion of the usefulness of a mathematical construction for physics. But the actual creative principle lies in mathematics. Thus, in a certain sense, I take it to be true that pure thought can grasp the real, as the ancients had dreamed
[4].

He did not touch, however, upon the basic question: How comes that mathematics is on one hand the brainchild of the human being and on the other hand is intrinsic in the framework of the universe? When he did refer to this question he admitted failure from the start "One may say "the eternal mystery of the world is its comprehensibility" [5].

It can be concluded, thus, that for Einstein, this question was solved once he accepted the philosophical world view and thus the "God" of Baruch-Benedictus Espinoza, who argued for the uniformity of the terms of "God"' and "Nature"2. Espinoza and thus Einstein, took it as granted that being a Supreme Mathematician is one of the infinite attributes of "God" i.e. Nature.

Eugene Wigner [6], not being a Spinozist, brought this enigma to the level of an absurd in the title in his paper "The Unreasonable Effectiveness of Mathematics in the Physical Science", in which he restated the problem by touching on the super-natural. His claim was that "The miracle of the appropriateness of the language of mathematics to the formulation of the laws of physics is a wonderful gift which we neither understand nor deserve." [6].

Indeed the achievements of the theories of Einstein, which were applauded by the world of science on the occasion of the hundred anniversary of Annum Mirabelis, had confirmed his trust in the power of mathematical thinking to unveil the many faces of nature.

One of the famous cases of forecasting, which was confirmed by many observations, is Einstein's General Theory of Relativity. In 1922 by developing the equations of this theory the Russian physicists Alexander Friedman [7] showed that these equations demand either a contracting or an expanding universe along space-time dimensions, which Einstein tried to stabilize by introducing his 'cosmological constant' [7]. Hubble's observations showed that this constant is redundant. Running back the "motion picture", namely turning expansion to contraction on space-time dimensions brought cosmologists to conclude that all started with a singular event, namely the Big Bang, after which the cosmos expands continuously to this day and into the future.

\section{The $5^{\text {th }}$ Dimension along Which Our Universe Expands}

All the computerized models used by the physicists to contract the universe to reach the pre-Big Bang singular point and expand it to its present dimensions and beyond were dictated by mathematical procedures, demanding

${ }^{2}$ Einstein's response the telegrammed question of New York's Rabbi Herbert S. Goldstein in (24 April 1929): "Do you believe in God? I believe in Spinoza's God, Who reveals Himself in the lawful harmony of the world, not in a God Who concerns Himself with the fate and the doings of mankind. 
various assumptions at various stages. The basic assumption, following Einstein's basic conceptual model is that our universe is four-dimensional, (three orthogonal spatial coordinate system and time which has only one degree of freedom from past to future). This continuum is measurable by an observer using a meter and a clock.

Yet, Einstein's, Friedman's and their colleagues, investigating the implication of the General Theory of Relativity, like the Dutch Willem de Sitter [8], and the French Georges Lemaitre [9], were all following the same laws of mathematics, which they assumed that the universe is following. However, it never occurred to these physicists to ask the following questions:

1) Along which dimension evolve the mathematicallogical structures they have constructed and were running in their brains and later on their computers.

2) Is it not possible that this sequence of ideas in their brain while thinking and trying to understand these theories is running along a dimension addition to space-time?

One will not be surprised, however, that any physicist adherent to the mandate of his profession i.e. investigating the physical world along the physical spatial-temporal dimensions, using a meter and a clock will revoke these questions, claiming that they belong to the field of 'meta-physics' and endangers the objective approach to science. In other words, once the subjective individual sitting in front of his computer becomes involved in the program being run on this objective machine, the results may be biased. This approach makes further questions redundant, like: The knowledge gained allowing the execution of a program by a computer every few years becoming faster than its previous ancestor, is it not due to progress in information (hardware and software)?

It goes without saying that we suggest that once such advancement is noticeable, it should be measured along a dimension of information.

Speaking about this additional dimension one comes to the 'fifth dimension' (i.e. the three spatial directions and the direction of time) introduced in by Kaluza in 1919 [10]. Although Kaluza was able to show that by introducing a fifth dimension then both gravity and electromagnetism can be described from the same underlying framework, and albeit Einstein's interest and preliminary acceptance [10], this $5^{\text {th }}$ dimension was not acceptable among physicists. The main reason, to the present authors' opinion, being that the $5^{\text {th }}$ dimension was a mathematical innovation and the physicists could not 'see it' 'measure it with their rulers' and clocks. No physicist, including Einstein, who pondered about the mystery of mathematics, dared to ask, how is it that a mathematical $5^{\text {th }}$ dimension is capable of unifying the electro-magnetic and the gravity fields. Is it not possible that the $5^{\text {th }}$ dimension is along the dimension of mathematics? Klein [10], a theoretical-physicist, proposed a solution to the physical deficiency in Kaluza's (1919) suggestion by attributing to the mathematical $5^{\text {th }}$ dimen- sion a spatial character of curling it up into a small enough space to escape ordinary detection.

Curling up dimensions and thus making them "physical", is a plausible solution from the point of view of the physicist, who configures with "four dimensional" universe. Yet his brain is free to roam multi-dimensional universes. In other words what Kaluza showed the physicists is that mathematics is a vehicle enabling to add dimensions and thus expanding beyond Space-Time.

The curling up of space, was also criticized by Hawking [11], in the case of discussing the multi dimensional (ten or twenty-six) space-time universe, suggested by the string theory $[12,13]$. His question is: Why should some, but not all, of the dimensions be curled up into a small ball? It is beyond the scope of the present article to discuss the answers that Hawking [11] suggests, the common factor of which is that these are all physical space-time dimensions. On the other hand the need to invent the conceptual model of the 'string theory' $[12,13]$ and add a non-observable dimension to the space-time continuum, may be regarded as a hint, what sort of a dimension it should be. More-overformulations accounting for Information as an additional dimension will also address the 11-dimensional M-theory [13] that requires space-time to have eleven dimensions.

\section{The Dimension not Accounted by Darwin}

While investigating the geology of the Quaternary, i.e. the layers deposited during the last two million years, of the Coastal Plain of Israel, the first author of the present article got acquainted with the evolution of the stone tools. These tools show evolution from primitive pebble tools, which were just pebbles etched at one end to become sharp and pointed, to the evolved flint arrow heads. In other words: a body showing a rise of spatial-complexity along the dimension of time. The evolution of this complexity expressed, as we know, also progress in the intelligence of its manufacturers. The question then arises whether this trend of evolution could be explained in the framework of the conventional Darwinian to NeoDarwinian paradigms, i.e. evolution through the process of random mutations filtered by the constraints of the hostile environment, which condemned to disappearance form the stage of existence the less fitted.

Thus, while success in the process of Darwinian selection, i.e. survival of the fittest, can be measured according to the number of similar forms of life and their distribution in space-time. The question which bothered the first author was: What about the ability of these forms of life who were able to change the hostile environment. As the ability to change the environment, in the case of the hominids, was a function of the evolution of their intelligence, the following question was: How should the survival of the more intelligent forms be measured? In other 
words, who are more successful from the point of view of the Darwinian paradigm, the hominids or the beetles?

The general question which follows is: By which units of measurements the evolution of intelligence can be quantified (when IQ tests are not feasible) and on what dimension can evolution of intelligence be measured?

Generalizing these questions, it can be said that the Darwinian paradigm explained beautifully the evolution of forms, i.e. spatial changes and the spread out of the forms along the space dimension as time passed, i.e. the time dimension. Now, at a certain point on the dimension of time the hominids branched off from the primates and started to produce tools. These tools became more and more sophisticated as time progressed and as the hominids multiplied and spread over the globe. The dimensions on which this progress on the tree of evolution is described, by conventional measures, are either temporal, i.e. the time passed since the first pebble-tools were produced, or spatial, i.e. the spatial features of the hominids and their tools, as well as their geographical range. All these data are along space-time dimensions and the question is: Once progress of intelligence became dominant in the evolutionary process should not an additional dimension be added to properly address this progress and evaluate it?

While the questions started with relation to hominids, in due time this question was generalized for the entire bio-world. This happened after coming upon the results of the research carried out by the psychologist Morton E. Bitterman [14], who found that the evolution of intelligent behavior in the bio-world correlates with the place of the species on the evolutionary tree. This meant that the increase of intelligence is parallel with the appearance of new forms of life on the geological timetable. Thus vertebrates are more intelligent than invertebrates, saurians than fishes, mammals than saurians, etc. Bitterman [14] investigated the level of intelligence by "the ability to develop a new way of reaction when an entirely new situation comes up." The question, which came up after reading this conclusion was whether there exists a dimension on which intelligence can be measured, except by the time needed to learn to push a button or find food in a maze? Moreover, once experience is gained and turned into instinctive behavior or abstract knowledge on what dimension can this be presented and evaluated, in addition to the spatial-temporal scales?

The answer to these questions is: The Dimension of Information”. Yet this dimension is not necessary just to describe the evolution of intelligence in the bio-world in general and that of the Homo sapiens-sapience in particular, but is also essential to describe the rise in the complexity of the physical world [15], namely the growth of the complexity of the structures composed of information bits, which means on one hand more information bits, as well as higher levels of organization of algebraic-logical sentences.
The above definition of Information actually describes the coordinates along which the intelligibility of a messages sent through any system of tele-communication can be measured either by telephone, telegraph, e-mail or internet. In this context it is generalizing the term of 'information' as defined in the theory developed by Shannon and Weaver [16]. In their theory they suggested that the loss in the intelligibility of a messages sent through any system of communication, namely its increasing distortion by 'noise', can be described in a similar formulation to Boltzman-Maxwell's formulation of the physical Second Law of Thermodynamics. Thus, one cannot avoid the general conclusion that the loss of meaning (decrease in the number of ordered sets of bits of information) of a certain message is through a process similar to that which determines the increase of entropy in a thermodynamic system. The addition of the Dimension of Information measured along the - information/time/space - dimensions, enables this loss of meaning to be expressed in physical-mathematical terms.

Physically-mathematically speaking, an increase in order is equated with increase in complexity and with organization, and is defined as negative entropy. Thus the Boltzman and Planck expression for the entropy of a system $S_{B P}=K \ln W$ (where $S_{B P}$ denotes the statistical entropy of a closed system, $K$ denotes Boltzman's constant and $W$ denotes the number of independent quantum states) can be regarded as strictly a thermodynamic statement.

On the other hand when the same expression is presented as $S_{B P}=-K \sum_{i=1}^{i=n} P_{i} \ln P_{i} \quad$ (where $P$ denotes the probability that the system exists in the microstate $i$ ) it becomes a measure of the probability of the system, i.e. the measure of our ignorance of the actual quantum state of the system. Such a measurement is also along the dimension of information, or more correctly, the exponential value on the dimension of information. This is proportional to the level of organization of the system. In other words, the more information and the higher is its exponential value so is the system of lower probability, and thus lowers entropy.

Instead of the expression for $S_{B P}$, a more general form [16] can be $I_{n}=-K \sum_{i=1}^{i=n} P_{i} \ln P_{i}$ where $I_{n}$ denotes the total information derived from a system where $\ln P_{i}$ expresses the total contributions of each subset of which the system is composed of, weighted by its probability.

In conclusion, the adding of the Dimension of Information enables to understand better the physical observation made by Shannon that the noise (reciprocal to the quantity of Information) in a communication system becomes greater the longer are the dimensions of spacetime. 


\section{A Few Words of Encouragement from Physicists}

Although we foresee the difficulties facing the undertaking of building a bridge between the space-time measurable world of the physicists and the one containing also the dimension of Information, still a blink of hope exists. This emerges from the fact that quantum physicists are becoming aware of the need to introduce the observer and an additional dimension, similar to that of Information, to their conceptual model in order to explain "bizarre" phenomena, in the micro-world. This can be seen in the following citations, which we believe can also be regarded as a support to the space-time-Information conceptual model:

1) "We have already considered with disfavour the possibility of the universe having been planned by a biologist or an engineer; from the intrinsic evidence of his creation, the Great Architect of the Universe now begins to appear as a pure mathematician.” [17].

2) "No, it's a new kind of wave which I call 'active information'. The notion of active information is already familiar to us from computers. Also, if I tell you something and you do something, that's obviously active information. If 'I shouted 'fire', everybody would move, so we know that in living intelligent systems, and in computers, active information is a useful concept. Now what I am proposing is that matter in general is not so different.” [1].

\section{Formulation}

As already mentioned physicists and mathematicians strive to formulate the multi-dimensional continuum in the framework of the conceptual model of the multi dimensional string theory. The following formulation is more modest and is exercised in the framework of the Hamiltonian extended stationary principle in terms of Space, Time and an additional degree of freedom, all as independent stationary variables. It is suggested that this degree of freedom may be regarded as an information state.

Thus, let us denote the information state by $I$, we postulate that similar to Hamilton's extended stationary principle, there exists a functional $\chi(f)$ being a function of its integrant $f$ between an initial information level $I_{0}$ and that of a final one $I_{f}$, in the form

$$
\chi=\int_{I_{0}}^{I_{f}} \int_{t_{0}}^{t_{f}} \int_{\Omega} f\left(I, t, \Omega, \phi, \phi_{I}, \phi_{t}, \phi_{\Omega}\right) d I d t d \Omega
$$

Note that in (1) we consider time $t\left(\in\left[t_{0}, t_{f}\right]\right)$ and space $\Omega$, to be independent of information. Accordingly, $\phi=\phi(I, t, \Omega)$ denotes the dependent function with its: information derivative $\phi_{I}(\equiv \partial \phi / \partial I)$, time derivative $\phi_{t}(\equiv \partial \phi / \partial t)$ and spatial derivative $\phi_{\Omega}(\equiv \partial \phi / \partial \Omega)$.

The spatial domain is assumed to be fixed. The necessary and sufficient condition to obtain minimum for $\chi$, is that the dependent function $\phi(I, t, \Omega)$ satisfy Euler's equation, namely

$$
\frac{\partial}{\partial I} \frac{\partial f}{\partial \phi_{I}}+\frac{\partial}{\partial t} \frac{\partial f}{\partial \phi_{t}}+\frac{\partial}{\partial \Omega} \frac{\partial f}{\partial \phi_{\Omega}}-\frac{\partial f}{\partial \phi}=0
$$

In what follows, we will investigate the implementation of the theory to a $1 \mathrm{D}$ problem.

\section{Example}

Let us consider a thin rod under vibrations along $x(\in[0, \ell])$ the longitudinal direction with $U=$ $U(I, t, x)$ as its wave amplitude.

For this proposed example, we relate $U$ with $\phi$, and choose $f$ in the form

$$
f=\frac{\mathcal{K}_{I}}{2}\left(\frac{\partial U}{\partial I}\right)^{2}+\frac{\mathcal{K}_{t}}{2}\left(\frac{\partial U}{\partial t}\right)^{2}+\frac{\mathcal{K}_{x}}{2}\left(\frac{\partial U}{\partial x}\right)^{2}+F U
$$

where $F=F(I, t, x)$ denotes the specific external driving force over a unit area, $\mathcal{K}_{I}, \mathcal{K}_{t}$ and $\mathcal{K}_{x}$ denote coefficients associated with the second partial derivative of $U$.

For example, let

$$
\left.\begin{array}{c}
\mathcal{K}_{I}=1 \\
\mathcal{K}_{t}=-1 / \vartheta^{2} \\
\mathcal{K}_{x}=1 / V^{2}
\end{array}\right\}
$$

where $\vartheta$ denotes the travelling speed of the information wave in the $I$-vs- $t$ plan and $V\left(=\frac{\vartheta}{C}\right)$ accounts for the ratio $\vartheta$ and $C$ the speed of a traveling wave in the $x$-vs- $t$ plan. In view of (1), (3) and (4) the functional $\chi$ to be varied, will be in the form

$$
\begin{aligned}
\chi=\int_{I_{0}}^{I_{f}} \int_{t_{0}}^{t_{f}} \int_{0}^{\ell}[ & \frac{(\partial U / \partial I)^{2}}{2}+\frac{(\partial U / \partial x)^{2}}{2 V^{2}} \\
- & \left.\frac{(\partial U / \partial t)^{2}}{2 \vartheta^{2}}-F U d I d t d x\right] \\
& +\int_{t_{0}}^{t_{f}} \int_{0}^{\ell}\left(Q U+Z U^{2} / 2\right) d t d x
\end{aligned}
$$

where $Q$ and $Z$ denote, respectively, generalized source and conductance terms on the boundary envelope in space and time.

Upon varying $\chi$, we describe an extremum process to define the $U$ function in $I, t, x$ terms that will minimize the $\chi$ functional. Assuming that the bound- 
ary conditions in (5) are fulfilled, we follow (2), Euler's equation, and obtain a modified wave equation in the form,

$$
\frac{\partial^{2} U}{\partial I^{2}}+\frac{1}{V^{2}} \frac{\partial^{2} U}{\partial x^{2}}-\frac{1}{\vartheta^{2}} \frac{\partial^{2} U}{\partial t^{2}}+F=0
$$

In what follows, we will consider different dominant forms that may be obtained from (6). To do this we will refer to nondimensional analysis.

\subsection{Dimensionless Analysis}

Let us denote the characteristic value of a property by ()$_{C}$. We choose the characteristic value so as to allow the dimensionless terms, [] ${ }^{*}$, be of a unit order. This will allow the comparison between the scalar factors multiplying the dimensionless terms.

The dimensionless form of (6) reads

$$
\begin{aligned}
{\left[\frac{\partial^{2} U}{\partial I^{2}}\right]^{*}+\frac{I_{C} C}{\vartheta L_{C}}\left[\frac{\partial^{2} U}{\partial x^{2}}\right]^{*} } & -\frac{I_{C}}{\vartheta \Delta t_{C}}\left[\frac{\partial^{2} U}{\partial t^{2}}\right]^{*} \\
& +\frac{I_{C}^{2} F_{C}}{U_{C}}[F]^{*}=0
\end{aligned}
$$

where $L_{C}$ denotes a characteristic spatial increment and $\Delta t_{C}$ denotes a characteristic time step. In view of (7) and the relative order of magnitude of its scalar numbers, we may obtain different dominant forms. To investigate these, let us also define:

$$
q \equiv \frac{L_{C}}{\Delta t_{C}}
$$

as a characteristic velocity,

$$
\alpha \equiv \frac{U_{c}}{\Delta t_{C}}
$$

as the characteristic amplitude rate,

$$
\rho_{I} \equiv \frac{I_{C}}{L_{C}}
$$

as the information density,

$$
\vartheta_{C} \equiv \frac{I_{C}}{\Delta t_{C}}
$$

as the characteristic traveling velocity of information, and

$$
\epsilon_{C} \equiv \frac{U_{C}}{L_{C}}
$$

as the characteristic aspect ratio.

\subsection{Traveling Wave in Space and Time}

Consider the possibility that,

$$
\left.\begin{array}{c}
\frac{\rho_{I}}{V} \gg 1 \\
\frac{\vartheta_{C}}{\vartheta} \gg 1 \\
\frac{\rho_{I}}{\epsilon_{C}} I_{C} F_{c} \gg 1
\end{array}\right\}
$$

In view of (13), the approximate form of (6) conforms to a traveling wave in the form,

$$
\frac{\partial^{2} U}{\partial x^{2}}-\frac{1}{C^{2}} \frac{\partial^{2} U}{\partial t^{2}}+V^{2} F=0
$$

Note that the driving force is amplified by the square of the ratio between traveling velocity of information and the velocity of the aforementioned traveling wave.

\subsubsection{Traveling wave in information and time} Consider the possibility that,

$$
\left.\begin{array}{c}
\frac{C}{q} \ll 1 \\
\frac{9 F_{c}}{\alpha} \ll 1
\end{array}\right\}
$$

In view of (13), the approximate form of (6) conforms to a traveling wave equation in the $I$-vs- $t$ plane

$$
\frac{\partial^{2} U}{\partial I^{2}}-\frac{1}{\vartheta^{2}} \frac{\partial^{2} U}{\partial t^{2}}+F=0
$$

It is worthy to stress that (16) occurs when the characteristic velocity is of greater magnitude than that of the traveling wave velocity and the ratio between the amplitude rate and the driving force be also of greater magnitude than the traveling velocity of the considered wave.

\subsubsection{Stagnation in Space and Information}

Consider the possibility that,

$$
\left.\begin{array}{c}
\frac{C}{q} \gg 1 \\
\frac{\vartheta F_{c}}{\alpha} \gg 1
\end{array}\right\}
$$

By virtue of (17) the dominant form of (6) will become,

$$
\frac{\partial^{2} U}{\partial I^{2}}+\frac{1}{V^{2}} \frac{\partial^{2} U}{\partial x^{2}}+F=0
$$

The amplitude surface which is obtained by the solution of (18), may exhibit craters and/or peaks, depending on the driving force which, as a function also of information, acts as a sink/source term.

\section{Questions and Conclusions}

The mathematician Kaluza in 1919 suggested that if the world was five dimensional ( 3 spatial + time $+5^{\text {th }}$ ) then electromagnetism and gravitation can be described by a 5 dimensional geometry. The physicist Klein [10] ex- 
plained the invisibility of this extra dimension, by adding to this geometric description, the principle of "perspective" namely that this dimension which we do not observe is because it is rolled up to a tiny size. Klein [10] computed the circumference of this tiny corpuscle to be about twenty powers of ten smaller than an atomic nucleus. The revolution of adding dimensions continued when the nuclear forces were discovered and the question rose whether to achieve a general theory these forces should not be incorporated into a Kaluza-Klein's [10] theory by this reducing all the forces of nature to pure geometry? This brought to the multi-dimensional string theory $[12,13]$ which brought to the formulation of the 11-dimensional M-theory [13]. Yet in the various articles discussing the physics of a multi-dimensional continuum, one can not find any suggestion of a dimension which is not spatial-temporal.

Focusing just on the period from Newton to Einstein to Kaluza-Klein [10] theories, every physicist will probably agree that there was evolution in the complexity of the physical conceptual model. Yet, the question, which should be asked, is: What about the evolution of human thinking, which became more complex since it had to address additional concepts. On what dimension did human thought evolve? Was it just on space-time?

The evolution of the biological sciences and especially that of the processes of heredity and genetics has even made these questions more relevant. Does the information contained in the DNA molecule described just by its space-time structure? Or just by its chemical configuration? Moreover, even if these descriptions are sufficient to pin-point a certain congenital trait, do they describe the past history of the evolution of these traits? These questions are now hotly debated, between the proponents of intelligent design, creationism, and Darwinism [18].

Generalizing these questions will be: Isn't it possible that there exists a non spatial-temporal dimension, which the physicists and biologists ignore because it is not observed, yet science on the whole is built and is continuously progressing along it? Our suggestion is: Indeed this is the Dimension of Information.

\section{References}

[1] P. C. W. Davies and J. R. Brown "The Ghost in the Atom Interviews with John Bell, David Bohm and Basil Hile,” Cambridge University Press, Cambridge, 1986, p. 128

[2] A. S. Issar, "Evolution of Intelligence in the Bio-World. Divine Action and Natural Selection: Questions of Sci- ence and Faith in Biological Evolution,” In: R. Gordon and J. Seckbach, Ed., World Scientific, Singapore, 2008.

[3] A. Einstein, "Geometry and Experience: Lecture before the Prussian Academy of Sciences on January 27, 1921," In: A. Einstein, Ed., Ideas and Opinions, Crown Publishers, New York, 1954.

[4] A. Einstein, "On the Method of Theoretical Physics,” In: A. Einstein, Ed., Ideas and Opinions, Crown Publishers, New York, 1954, pp. 270-276.

[5] A. Einstein, "Physics and Reality," Franklin Institute Journal Ditto, 1936, pp. 290-322.

[6] E. Wigner, "The Unreasonable Effectiveness of Mathematics in the Natural Sciences," Communications on Pure and Applied Mathematics, Vol. 13, No. 1, 1960, pp. 1-14.

[7] A. Friedman, "English Translation in: On the Curvature of Space," General Relativity and Gravitation, Springer Netherlands, Vol. 31, 1999. Original article in: T. Kaluza, “Über die Krümmung des Raumes," Zeitschrift für Physik, Vol. 10, No. 1, 1999, pp. 377-386.

[8] P. Kerszberg, "The Invented Universe: The Einstein-De Sitter Controversy 1916 and the Rise of Relativistic Cosmology,” Oxford University Press, 1989.

[9] J. Farrell, "The Day without Yesterday: Lemaitre, Einstein and the Birth of New Cosmology," Thunder's Mouth Press, New York, 2005.

[10] P. S. Wesson, "Five-Dimensional Physics: Classical and Quantum Consequences of Kaluza-Klein Cosmology," World Scientific Publishing, Singapore, 2006.

[11] S. W. Hawking, "A Brief History of Time-From the Big Bang to Black Holes,” A Bantam Book, 1988.

[12] J. Polchinski, "String Theory," Cambridge University Press, Cambridge, 1998.

[13] M. Kaku, "Strings, Conformal Fields, and M-Theory," Springer-Verlag, New York, 1999.

[14] M. E. Bitterman, "Phyletic Differences in Learning," American Psychologist, Vol. 20, 1965, pp. 396-410.

[15] S. A. Kauffman, "The Origins of Order," Self Organization and Selection in Evolution, Oxford University Press, New York, Oxford, 1993.

[16] W. Weaver and C. E. Shannon, "The Mathematical Theory of Communication," University of Illinois Press, Urbana, 1963.

[17] H. J. Jeans, “The Mysterious Universe,” Cambridge University Press, Cambridge, 1930, p. 134.

[18] A. S. Issar, "Evolution of Intelligence in the Bio-World Divine Action and Natural Selection: Questions of Science and Faith in Biological Evolution,” In: R. Gordon and J. Seckbach, Eds., World Scientific, Singapore, 2009. 